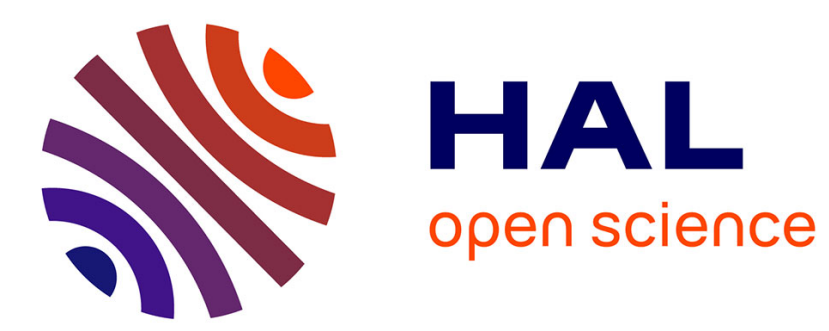

\title{
Roger Caillois dans les impasses du Collège de Sociologie
}

Guillaume Bridet

\section{To cite this version:}

Guillaume Bridet. Roger Caillois dans les impasses du Collège de Sociologie. Littérature, 2007, 146, pp.90-103. 10.3917/litt.146.0090 . halshs-01529928

\section{HAL Id: halshs-01529928 \\ https://shs.hal.science/halshs-01529928}

Submitted on 31 May 2017

HAL is a multi-disciplinary open access archive for the deposit and dissemination of scientific research documents, whether they are published or not. The documents may come from teaching and research institutions in France or abroad, or from public or private research centers.
L'archive ouverte pluridisciplinaire HAL, est destinée au dépôt et à la diffusion de documents scientifiques de niveau recherche, publiés ou non, émanant des établissements d'enseignement et de recherche français ou étrangers, des laboratoires publics ou privés. 


\section{Roger Caillois dans les impasses du Collège de Sociologie}

Le Collège de Sociologie, dont la déclaration inaugurale paraît en juillet 1937 dans la revue Acéphale, est à maints égards une « institution étrange, résolument en marge des formes classiques d'enseignement et de diffusion du savoir » ${ }^{1}$ et éloignée, plus largement, de tout ce qui se donne à entendre et à lire dans la France intellectuelle et politique de la fin des années 1930. Loin de chercher à accentuer cet isolement, le Collège prend cependant place au cœur des grands bouleversements de son époque dans le double cadre d'une faiblesse des démocraties libérales incapables de répondre aux agressions des régimes fascistes, mais aussi d'une impossibilité des écrivains d'avant-garde à se mêler efficacement aux luttes politiques. Bataille, Caillois, Leiris : les trois animateurs principaux du Collège sont d'anciens surréalistes qu'à l'heure de la montée des périls a fini par agacer un verbiage poétique qu'ils jugent sans la moindre prise sur la réalité. L'idée est alors de se tourner vers la science et, plus précisément, vers cette discipline apparue à la fin du XIX ${ }^{e}$ siècle, qui a pour nom sociologie et qui, dans les années 1930, souffre encore d'une certaine fragilité institutionnelle mais qui attire des jeunes gens énergiques ${ }^{2}$. Les trois hommes font alors le pari - mais pour des raisons différentes qui vont rapidement conduire à des divergences de fond - que la jeune sociologie française peut apporter à la littérature ce qui lui manque : de l'universalité, de la nécessité et donc une puissance d'action politique et sociale. Ce passage de la volonté de connaissance à la volonté de puissance, qui déroge au respect d'une certaine neutralité scientifique, est bien entendu mal reçu par la sociologie institutionnelle française. Mais il conduit plus gravement, dans l'optique même de Caillois, à une impasse : le verbiage poétique cède en effet la place à un échauffement rhétorique que caractérise un verbalisme fait de virilité affichée et de prophétisme

1. Bruno Karsenti, L'Homme total. Sociologie, anthropologie et philosophie chez Marcel Mauss, Paris, PUF, 1997, p. 377. hautes études dans les années 1930. Quant à Bataille, il a découvert ses travaux par le biais d'Alfred Métraux, son condisciple à l'École des Chartes qui fut aussi l'élève de Mauss dans les années 1920. 
menaçant mais qui ne produit nul effet social. Un rêve s'efface — qui est finalement d'un poète français accordant trop de crédit aux mots.

Prenant place dans le champ de la recherche sociologique, le Collège se veut d'abord un institut consacré à «l'étude de l'existence sociale dans toutes celles de ses manifestations où se fait jour la présence active du sacré $»^{3}$. L'objet privilégié qu'il se choisit le place très clairement dans la tradition de la sociologie durkheimienne qu'animent une exigence de communion et un besoin de loi. D'un côté, l'individu n'existe que par le groupe auquel il appartient et il ne peut vivre en dehors d'un encadrement; de l'autre, la cohésion sociale trouve son fondement dans une communion normative fondée sur des croyances partagées. Mauss, chargé par Durkheim de s'intéresser aux études religieuses dans les sociétés archaïques, se montre sur ce point fidèle à l'enseignement de son maître et partage lui aussi l'idée que la religion est le fait intégrateur essentiel de la société en ce qu'elle détermine les jugements de valeur que partage chacun de ses membres.

$\mathrm{Si}$ le sacré constitue bien la thématique centrale commune au Collège de Sociologie et à l'école sociologique française, le premier n'entend cependant pas s'arrêter à la seule considération des faits sociaux mais «établir les points de coïncidence entre les tendances obsédantes fondamentales de la psychologie individuelle et les structures directrices qui président à l'organisation sociale et commandent ses révolutions ${ }^{4}$.

Cette prise en compte de déterminations psychologiques « fondamentales », c'est-à-dire fondées sur la réalité biologique de l'homme, constitue une rupture majeure avec la tradition sociologique française. Les phénomènes sociaux qu'envisage en effet Durkheim «ne sauraient se confondre avec les phénomènes organiques, puisqu'ils consistent en représentations et en actions, ni avec les phénomènes psychiques, lesquels n'ont d'existence que dans la conscience individuelle et par elle » ${ }^{5}$. La sociologie n'envisage les faits psychologiques qu'en tant qu'ils sont le produit de déterminations sociales indépendantes de la volonté individuelle et douées d'une puissance impérative et coercitive qui s'impose à elle. Pour ce qui est de l'être biologique de l'homme, celui-ci est en tout point opposé à son être social. Il y a, d'un côté, les sensations et les tendances sensibles, de l'autre, la pensée conceptuelle et l'action morale. Les premières, égoïstes et individuelles, sont liées à notre organisme ; les secondes, susceptibles d'être universalisées et communément partagées, nous viennent au contraire de la société. Entre ces deux réalités humaines, il y a un véritable antagonisme qui interdit de penser que puisse entrer

3. Roger Caillois, «Introduction », [NRF, $\mathrm{n}^{\circ} 298$, juillet 1938], repris in Denis Hollier, Le Collège de Sociologie (1937-1939), [1979], Paris, Gallimard, 1995, p. 300.

4. Ibid.

5. Émile Durkheim, Les Règles de la méthode sociologique, [1895], Paris, PUF, 1990, p. 6. 
dans la détermination spécifique du fait social quoi que ce soit de strictement lié à la biologie de l'espèce.

En définissant son domaine de recherche au croisement d'une discipline qui prendrait en compte les déterminations individuelles et naturelles et les déterminations collectives, le Collège se trouve conduit à la pratique d'une interdisciplinarité sauvage mêlant psychanalyse, biologie et sociologie et il se rend donc coupable d'une transgression majeure par rapport à la manière dont est constitué le champ de la sociologie française. La sociologie a pour but d'expliquer les faits sociaux par d'autres faits sociaux et non par des faits relevant d'autres domaines de l'investigation scientifique. Les reproches que, en bon disciple de Durkheim, Mauss adresse à Caillois à la suite de la parution du Mythe et l'homme sont de ce point de vue fort clairs : «Quant à votre biologie générale, elle appelle les réserves les plus fortes. [...] la philosophie de la biologie n'a aucun rapport avec la philosophie de la société. ${ }^{6}$

Il faut ajouter - seconde transgression - que les buts que se propose d'atteindre le Collège ne sont pas uniquement de nature scientifique. Ils sont aussi moraux et politiques. Atteindre « la compréhension de l'ensemble des phénomènes sociaux » est certes d'abord, comme l'explique Caillois dans son texte inaugural, le moyen de déterminer «les maximes d'action qui trouvent en elle référence et garantie ${ }^{7}$. Si le Collège n'avait prétendu qu'à comprendre la société contemporaine afin de déterminer plus sûrement des choix politiques, il serait resté proche des perspectives de Mauss et de Durkheim. Le projet d'une science sociale est en effet lié en France, dès son origine, dans le contexte d'un enfantement difficile de la III ${ }^{e}$ République, à un souci profond d'action. Contre Max Weber qui affirme la nécessité d'une séparation entre le savant et le politique, Durkheim lie indissociablement le métier de sociologue à la solidification des institutions républicaines. « Nous estimerions que nos recherches ne méritent pas une heure de peine si elles ne devaient avoir qu' un intérêt spéculatif ${ }^{8}$, déclare-t-il ainsi. Sensible au réformisme socialiste, il considère qu'il convient de lui adjoindre une sociologie scientifique qui puisse se démarquer des idéologies et apporter une réponse crédible au dérèglement des sociétés industrielles. Pour Durkheim, la sociologie est, comme le dit justement Denis Hollier, « une éducation civique $»^{9}$. Le sociologue ne doit pas faire une carrière proprement politique mais, plus modestement, être un conseiller, un éducateur et agir par le livre, la conférence ou les œuvres d'éducation populaire. Sous ce rapport, Mauss n'est qu'apparemment éloigné de lui. Certes, il reste

6. Marcel Mauss, lettre à Roger Caillois, 22 juin 1938, reprise in Roger Caillois, Paris, 
toute sa vie un militant socialiste et s'implique durablement dans le mouvement coopératif, mais ces engagements publics ne le poussent pas à revendiquer, dans l'exercice même de sa recherche, une position de pouvoir politique. Le politique doit beaucoup au savant dans la mesure où le savoir qu'il détient guide son action; le savant doit en revanche préserver son indépendance par rapport au politique.

Tel n'est pas le cas des membres du Collège de Sociologie qui prétendent participer à la vie politique dans le cadre même de leur activité sociologique en s'appuyant sur « le caractère nécessairement contagieux et activiste des représentations » qu'ils étudient pour fonder « une communauté morale, en partie différente de celle qui unit d'ordinaire les savants et liée précisément au caractère virulent du domaine étudié et des déterminations qui s'y révèlent peu à peu ${ }^{10}$. L'entreprise implique certes « un sérieux, un désintéressement, une sévérité critique » qui sont liés au respect d'une morale professionnelle, mais ces qualités, loin de viser seulement à « accréditer les résultats éventuels », ont pour but ultime d' « imposer le respect ${ }^{11}$. Comme le note à la même époque Caillois, la notion de respect est intimement liée à celle de sacré et toutes deux constituent les attributs essentiels du pouvoir. Le caractère activiste de la sociologie n'est ainsi pas le fruit d'une décision arbitraire ; il est fondé sur un objet d'étude particulier qui la contamine en retour. C'est en suivant la même logique que Caillois finit par faire allusion à Nietzsche et qu'il évoque « l'ambition que la communauté ainsi formée déborde de son plan initial, glisse de la volonté de connaissance à la volonté de puissance, devienne le noyau d'une plus vaste conjuration ${ }^{12}$. Caillois, Bataille et Leiris présentent le Collège comme un foyer d'énergies ayant vocation à réunir une élite autoproclamée asseyant son pouvoir sur l'ensemble de la société et assurant son renouveau. Il s'agit de passer de la sociologie du sacré à la sacralisation des sociologues - elle-même débouchant sur une resacralisation de la société. Reste qu'en affirmant la nécessité d'une théorie qui serait directement une pratique politique, le Collège remet en cause la séparation de la connaissance et de l'action et transgresse une règle majeure de l'activité scientifique.

Mais qu'est-ce qui pousse un sociologue de formation comme Leiris, un tout récent normalien et agrégé de grammaire comme Caillois et un ancien chartiste comme Bataille, à transgresser ainsi les règles de la méthode sociologique qu'ils connaissent pourtant fort bien? Cette volonté activiste du Collège ne peut se comprendre si on ne la rapproche pas de l'échec des mouvements et des revues d'avant-garde éphémères qui, de Contre-Attaque à Inquisitions puis à Acéphale, se développent dans le sillage du surréalisme dans la seconde moitié des années 1930.

10. Roger Caillois, «Introduction », op. cit., p. 299.

11. Ibid., p. 300.

12. Ibid., p. 301 
Ces revues et ces mouvements prennent naissance dans le contexte particulier de ce que certains, comme Char ou Crevel, considèrent comme une dérive esthétisante du surréalisme à un moment où le contexte politique requiert au contraire toute l'attention. Certes, comme l'écrit luimême Caillois, faisant allusion aux mésaventures des surréalistes avec le Parti communiste, «les écrivains » voient « leurs préoccupations intimes si mal s'accorder aux exigences de leur cause qu'ils [doivent] rapidement se soumettre ou se démettre ${ }^{13}$. Mais Caillois ne cherche pas à expliquer cet échec par la particulière rigueur du Parti communiste sous l'influence d'un Komintern confondant alors matérialisme historique et propagande au service de la lutte des classes. Si le surréalisme a échoué à s'imposer sur le terrain politique, c'est, selon lui, pour des raisons qui ne tiennent pas à la conjoncture mais à sa nature littéraire elle-même. Comme il le laisse déjà entendre dans La Nécessité d'esprit et comme il le constate encore dans Le Mythe et l'homme, la littérature explore de manière trop individualiste l'intériorité humaine pour mettre socialement en branle les forces profondes qui l'animent. «Les formes avancées de la littérature et de l'art », parmi lesquelles il faut compter « le surréalisme », ne proposent que «des activités semi esthétiques» débouchant sur «un caractère maniaque et purement rituel ${ }^{14}$, entendons d'un rituel qui n'a rien de collectif et se trouve réduit au petit groupe de ses sectateurs.

Caillois n'est pas sans faire preuve ici de quelque injustice à l'égard de Breton qui, à y regarder de près, estime lui aussi à la même époque que la pensée et la poésie n'ont de valeur que si elles sont susceptibles de motiver une action dans le cadre global de la société. Mais il est vrai que la contrainte que Breton s'impose de dialoguer avec le marxisme et avec le Parti communiste ne lui facilite pas la tâche dans la mesure où elle lui impose de batailler contre une soumission pratique de la pensée et de la poésie à la propagande et contre leur détermination théorique par l'infrastructure économique et sociale. C'est dans ce contexte d'un rapport complexe entre avant-garde politique et avant-garde poétique et dans le souci de son dépassement que les trois principaux membres du Collège, tous dissidents du surréalisme, vont chercher dans l'idéalisme de la tradition sociologique française un contre-feu idéologique contre le matérialisme marxiste permettant, non pas seulement de préserver une autonomie de la sphère de la pensée par rapport à la réalité économique et sociale, mais d'affirmer la supériorité de la première sur la seconde - manière de se démarquer de Breton mais aussi de franchir une étape de plus sur le chemin de l'illusion.

Contre la sociologie marxiste, qui pose le primat matérialiste des rapports de production et de la lutte des classes, Mauss, fidèle en cela à la

13. Ibid., p. 297.

14. Roger Caillois, Le Mythe et l'homme, [1938], Paris, Gallimard, 1987, p. 177-178. 
pensée de Durkheim et de Simiand, affirme lui-même le primat du symbolique sur l'économique : «Les faits sociaux sont [...] des causes parce qu'ils sont des représentations ou agissent sur des représentations. Le fond intime de la vie sociale est un ensemble de représentations. » ${ }^{15}$ Caillois ne dit pas autre chose quand, s'appuyant sur l'Essai sur le don de son maître, il critique le réductionnisme économique du marxisme. À ses yeux, « les phénomènes primitifs » ne relèvent « aucunement de la raison ou de l'intérêt bien entendu » mais sont au contraire «presque entièrement fonction des croyances religieuses, de l'organisation sociale ${ }^{16}$. Au marxisme qui pose la détermination en dernière instance de la réalité sociale et politique mais aussi de la conscience par l'infrastructure économique, au surréalisme qui essaie de faire la part des choses entre détermination sociale et création poétique, Caillois préfère la sociologie française qui pose le primat de la dimension symbolique sur tout autre type de déterminations. Il propose donc, comme les autres membres du Collège, une sociologie pour poète: sans infrastructures économiques ni classes sociales ni intérêts divergents parce que, garantissant la toute-puissance idéaliste de la pensée sur les individus comme la dimension communielle du social, elle est la seule à fonder théoriquement la mise en avant d'une élite spirituelle capable de produire et d'imposer à l'ensemble de la société ses représentations et ses valeurs.

C'est dans ce contexte d'une crise des démocraties redoublée par la difficulté de la littérature à avoir prise sur elle que les mythes font sentir leur importance particulière aux yeux des membres du Collège de Sociologie comme à de nombreux intellectuels et écrivains des années 1930 on pense à des individus aussi variés qu'Antonin Artaud, Denis de Rougemont ou Georges Dumézil. La démocratie libérale, fondée sur les principes de l'égalité et de la liberté, est par essence le régime de l'affaiblissement (individuel) et de la dispersion (collective) des forces. Appuyé sur une commune appartenance biologique à l'espèce garante de certaines réactions psychologiques élémentaires, le mythe est une réalité collective, c'est-à-dire sociale — on retrouve ici l'éclectisme du Collège entre sociologie, biologie et psychanalyse —, et il témoigne de la puissance du symbolique. Le mythe est une représentation qui s'impose à tous les individus sans être discutée et qui fonde les valeurs sur lesquelles reposent les sociétés. Il est donc susceptible de restaurer la communion sociale perdue. La littérature n'est que la dégradation du mythe. Ce dernier, qui a « autorité » et « force coercitive », se transforme en effet en « littérature » quand il perd «sa puissance morale de contrainte » et n'est plus qu' « objet de

15. Marcel Mauss, «Divisions et proportions des divisions de la sociologie », [Année sociologique, nouvelle série, n 1, 1927], Euvres, t. 3, Paris, Éditions de minuit, 1969, p. 160.

16. Roger Caillois, «L'Économie primitive, par Richard Thurnwald (Payot) », [Les Cahiers du Sud, $\mathrm{n}^{\circ}$ 199, novembre 1937], Roger Caillois, Jean-Clarence Lambert (dir.), Paris, Éditions de la différence, 1991, p. 58. 
jouissance esthétique ${ }^{17}$ pour quelques âmes perdues. Animé par le désir d'en finir avec la grande dualité qu'instaure la pensée moderne entre la pratique et la théorie et mêlant les motifs idéologiques contradictoires d'un dépassement de la littérature par la science et d'un retour vers l'archaïsme du mythe, le Collège utilise ainsi la sociologie universitaire du premier tiers $\mathrm{du} \mathrm{XX}^{\mathrm{e}}$ siècle afin de rédimer l'impuissance de mouvements littéraires d'avant-garde trop individualistes et subjectifs, et de satisfaire le désir activiste d'une toute-puissance de la chose écrite sur les choses du monde.

La prétention élitiste des poètes sociologues à recréer de la communion sociale au moyen d'une étude des mythes et du sacré qui aurait une capacité de contamination ne va cependant pas sans un idéalisme qui n'est pas seulement théorique mais aussi pulsionnel.

Fidèle à son principe aristocratique, Caillois postule ainsi «l'opposition éthique essentielle d'au moins deux classes d'êtres » ${ }^{18}$ fondée en nature. Afin de caractériser cette opposition, il substitue à l'opposition nietzschéenne entre les Maîtres et les Esclaves un couple de mots emprunté à Saint-Simon et qu'il juge en rapport plus étroit avec la réalité contemporaine. Le consommateur est «un type d'hommes [...] improductifs par eux-mêmes, digérant seulement, parasites d'autrui »; le producteur a au contraire «le monopole des audaces et des initiatives » et il produit « les mœurs » et « les usages $»{ }^{19}$. Caillois se livre ici à une relecture de la fin de l'Essai sur le don dans lequel Mauss, étendant ses observations sur les systèmes d'échange des sociétés primitives à ceux des sociétés modernes, en appelle, contre « une morale de marchand», à une restauration «des mœurs de "dépense noble" » ${ }^{20}$. Il radicalise cependant cette proposition en rompant la réciprocité de ce que Mauss appelle «le système des prestations totales ${ }^{21}$ et en transformant le don en une lutte dont l'enjeu devient l'acquisition du bien social et symbolique qu'est le prestige. Le potlatch sans réciprocité devient ainsi le fondement d'une réactivation violente du lien social ${ }^{22}$. Du côté du producteur, se trouve une force active et féconde qui peut légitimement revendiquer le pouvoir de contrainte, du côté du consommateur, une faiblesse passive et repue dont l'unique mode d'être est l'obéissance.

17. Roger Caillois, Le Mythe et l'homme, op. cit., p. 154.

18. Roger Caillois, «Le Vent d'hiver », [NRF, $\mathrm{n}^{\circ} 298$, juillet 1938], repris in Denis Hollier, Le Collège de Sociologie (1937-1939), op. cit., p. 339.

19. Ibid., p. 345-346.

20. Marcel Mauss, «Essai sur le don. Forme et raison de l'échange dans les sociétés archaïques », [Annales sociologiques, seconde série, 1923-1924, t. 1], Sociologie et anthropologie, [1950], Paris, PUF, 1980, respectivement p. 258 et p. 262.

22. On est ici dans une perspective proche des Réflexions sur la violence de Sorel, auteur auquel Caillois s'intéresse, comme en témoigne une note de lecture qu'il publie en avril 1936 dans la $N R F$. 
Seule la négation du corps réel du producteur autorise cependant l'affirmation d'une toute-puissance de sa pensée - négation qui se lit dans l'affirmation d'une parfaite autonomie. Influencé par la conception du pouvoir telle qu'elle est exposée en 1935 par Dumézil dans Ouranos-Varuna. Étude de mythologie comparée indo-européenne, Caillois explique que c'est uniquement en renonçant à tous ses appétits que le clerc donne "l'irréfutable preuve de sa haute qualité d'âme » et s'approprie « un droit essentiel sur ceux qui se contentent de ce qu'il dédaigne ${ }^{23}$. Appétit sexuel, appétit de gloire, de richesse ou même de pouvoir : le renoncement volontaire à toute forme de satisfaction — de jouissance - se renverse en un constat de supériorité qui fonde la légitimité du pouvoir et qui interdit en même temps tous les abus visant à satisfaire un désir personnel. La légitimité du pouvoir passe ainsi par la maîtrise morale de son propre désir avant d'envisager celle, politique, du désir de l'autre. Le corps du producteur est un corps idéal qui n'est pas soumis aux exigences de la nature : il n'a ni désir ni même besoin — insupportables dépendances. Dans «L'aridité », Caillois écrit qu' « il est des dénuements qui rendent plus puissants que l'opulence » et il loue «la stricte sobriété de l'orgueil » ${ }^{24}$. Résister à l'appétit et à l'aboutissement que constitue le plaisir, c'est faire l'échange d'un corps ouvert aux échanges et donc dégradé contre un autre, pur et glorieux, parfaitement homogène, n'ayant recours à aucun carburant et ne laissant aucune place aux dissensions intestines. C'est cet accroissement de l'être aux dépens de l'avoir qui fonde le principe d'échange énergétique interne substituant la toute-puissance magique de la pensée à la faiblesse du corps. Caillois affirme ainsi que «les valeurs » promues par les clercs sont «autant de projections agissantes » ${ }^{25}$ qui transforment et ordonnent le monde selon leurs désirs en prolongeant au-dehors l'ordre qu'ils ont fait triompher en eux-mêmes. Elles sont encore «les véritables idées qui mènent le monde » ${ }^{26}$. Le producteur social s'assure « le consentement intime » ${ }^{27}$ des consommateurs, leur impose ses valeurs et ses représentations et détermine donc, de l'intérieur, leurs actions.

Les trois derniers paragraphes du «Vent d'hiver» constituent sans aucun doute le passage le plus exemplaire de cette tentative d'exercer un contrôle social par la seule pensée. Si on laisse de côté l'interprétation politique de la métaphore finale, particulièrement ambiguë puisqu'elle appelle de ses vœux des «vents meurtriers et si salubres »

$\overline{23 .}$ Roger Caillois, «Sociologie du clerc», [NRF, $\mathrm{n}^{\circ} 311$, août 1939], Approches de l'imaginaire, Paris, Gallimard, 1974, p. 67-68.

24. Roger Caillois, «L'aridité », [Mesures, n³, avril 1938], Naissance de Lucifer, Montpellier, Fata Morgana, 1992, respectivement p. 63 et p. 64.

25. Roger Caillois, « Sociologie du clerc », op. cit., p. 69.

26. Ibid.

27. Roger Caillois, «L'aridité », op. cit., p. 67. 


\section{- réfleXions Critioues}

qui tuent «les délicats» et laissent le terrain libre pour « les plus aptes ${ }^{28}$, on peut noter qu'on est en présence d'une profération magique censée transformer la société et opposée, dans le texte lui-même, aux paroles inutiles parce qu'inefficaces. «Les oiseaux» comme «les beaux parleurs » ont une voix séductrice mais beaucoup trop faible pour se faire entendre « des oreilles sourdes aux chansons, mais vigilantes et exercées » qui sont celles des hommes forts ; «le champ est libre pour les plus aptes, poursuit Caillois : [...] nul gazouillis mélodieux et innombrable pour couvrir leur voix $»{ }^{29}$. Ce champ libre, qui est d'abord la société dont la secte a vocation à prendre la direction, est aussi et avant tout le chant libre, la musique du texte lui-même. Ce qui relève de la mélodie est assimilé à de la faiblesse et congédié au profit d'un autre usage de la langue, créateur de la réalité sociale comme de la réalité individuelle. Caillois s'en explique clairement dans une lettre à Paulhan :

Moi qui déteste les phrases, j'ai essayé d'en faire, dans la pensée qu'il se trouverait peut-être des gens qui seraient gagnés par l'atmosphère et feraient nombre, rendant cette atmosphère effective. [...]. Vous savez, je ne suis pas écrivain, il m'intéresse seulement d'avoir une action. ${ }^{30}$

Il s'agit à la fois, par l'écriture et la publication de ce texte, de se transformer soi-même en même temps qu'on transforme la société, de conduire une expérience imaginaire singulière mais qui pourrait devenir commune à tous les imaginaires et déterminer l'action de chacun. Contre tout principe de réalité, Caillois se donne une image idéale de lui-même qui l'apparente à un monarque thaumaturge capable magiquement, par la seule déclamation de formules choisies, de soigner le corps malade de la société.

Pour quel résultat? Alors qu'en Argentine, vers laquelle il s'est embarqué en juin 1939, Caillois décide de poursuivre les activités du Collège pour finalement les interrompre en 1941, à Paris le groupe qui se reconstitue autour de Bataille a une existence encore plus éphémère. La fin de l'entreprise du Collège s'explique d'abord par l'éloignement physique de ses membres; elle témoigne aussi d'un éloignement théorique qui ne fait que renvoyer aux apories de l'activisme sociologique dont Caillois est le plus ardent défenseur.

28. Roger Caillois, «Le vent d'hiver», op. cit., p. 352-353. Voir la mise au point de Denis Hollier, « De l'équivoque entre littérature et politique », Les Dépossédés (Bataille, Caillois, Leiris, Malraux, Sartre), Paris, Les Éditions de Minuit, 1993, p. 109-130. 
En lieu et place d'une maîtrise de soi quasi ascétique, Caillois se trouve d'abord obligé de constater que la Seconde guerre mondiale occasionne un déchaînement instinctuel qu'aucun contrôle de soi ne vient entraver. Sur ce point encore, Mauss ne se prive du reste pas de critiquer son jeune élève dès la fin des années 1930 et il met en cause «cette espèce d'irrationalisme absolu [...], probablement sous l'influence de Heidegger Bergsonien attardé dans l'hitlérisme, légitimant l'hitlérisme entiché d'irrationalisme ${ }^{31}$. C'est également sur ce point que Caillois et Bataille en viennent finalement à s'opposer. La volonté de puissance telle que l'entend le premier n'est en effet pas identique à «cette "avide volonté d'être" » 32 invoquée par le second et qui désigne tout au contraire l'exercice d'une souveraineté sacrificielle. Là où Caillois se défait de toute la faiblesse humaine en la projetant sur autrui et entend fonder un pouvoir spirituel sur l'affirmation d'une supériorité morale et d'un ordre homogène de conjurés, Bataille renvoie au contraire chaque individu à sa propre tragédie et la communauté à l'hétérogénéité de ce qui l'ouvre et l'unifie : la mort, le sacrifice ou la dépense. Tout se noue autour de leur lecture respective de Nietzsche. C'est du point de vue de la tragédie, et non du point de vue aristocratique de la maîtrise de soi, que Bataille critique le fascisme comme théorie politique conduisant à une suppression et non à une valorisation de l'hétérogénéité et de la souillure, alors que lui-même expose la nécessité d'une souveraineté qui accepterait son identification tragique avec elles. Si cette différence n'empêche pas Caillois de reporter systématiquement a posteriori et avec une certaine mauvaise foi la responsabilité de son rêve de puissance sur le seul Bataille, il n'en fait pas moins aussi son autocritique en reprenant les termes de la déclaration inaugurale du Collège :

La guerre vint qui, avec une force et à une autre échelle, se chargea alors selon notre programme «d'établir les points de coïncidence entre les tendances obsédantes fondamentales de la psychologie individuelle et les structures directrices qui président à l'organisation sociale et commandent les révolutions. ${ }^{33}$

En dissipant aux yeux de Caillois l'illusion d'une libération sous contrôle des instincts dans le cadre d'une secte des purs, la guerre rompt l'équilibre de la volonté de puissance et du renoncement ascétique. L'histoire objective dans la barbarie nazie l'absence de dimension sacrificielle et la présence unique de la domination d'autrui aux dépens de la domination de soi.

31. Marcel Mauss, lettre à Roger Caillois, 22 juin 1938, op. cit., p. 205.

32. Georges Bataille, «L'apprenti sorcier», [NRF, $\mathrm{n}^{\circ} 298$, juillet 1938], repris in Denis Hollier, Le Collège de Sociologie (1937-1939), op. cit., p. 319.

33. Roger Caillois, « Notes pour un itinéraire de Roger Caillois », Roger Caillois, op. cit., p. 168. 
L'idéal activiste de Caillois achoppe cependant aussi pour des raisons qui lui sont propres et qui ne concernent pas la réalité extérieure de la guerre. Les producteurs du Collège sont en effet bien en mal de fournir aux consommateurs les représentations collectives censées assurer la cohésion contraignante de la société autour de valeurs et d'actions communes. Telle est la critique essentielle que Leiris adresse au Collège. S'il ne donne qu'une seule conférence sur «Le sacré dans la vie quotidienne » en janvier 1938 et s'il garde une position de retrait par rapport à ses activités, ce n'est pas seulement que « des fautes très graves contre les règles de la méthode établie par Durkheim ${ }^{34}$ ont été commises. La publication de L'Afrique fantôme en 1934 indique bien que lui-même est suffisamment éloigné du professionnalisme des ethnologues pour ne pas s'en réclamer absolument. Ce qu'il ne peut en revanche supporter, c'est la fondation d'une communauté morale avec «des gens issus du milieu intellectuel» et qui ne peut donc que ressusciter «les pires formes des chapelles littéraires ${ }^{35}$. Pour celui qui parlera bientôt de la littérature comme d'une tauromachie, la prétention du Collège et particulièrement de Caillois à confondre réflexion et action est pure vacuité et n'est en rien supérieure aux vaines prétentions surréalistes. Décrire une réalité sociale ne suffit pas à la faire advenir. L'étude des mythes n'est pas à même de redonner à la société la cohésion qui lui manque. Après la guerre, Caillois se fait l'écho de ces propos et il se livre là encore à une autocritique sévère et répétée. Il rappelle ainsi à l'occasion de la réédition de L'Homme et le sacré en 1963 qu'il imaginait "pouvoir transformer un ardent savoir en un levier tout-puissant», mais que « contagion vertigineuse » et «effervescence épidémique » n'étaient que de «creuses ambitions » et restèrent « lettre morte ${ }^{36}$.

Si cette autodépréciation est à la mesure de la déception éprouvée devant l'impossible mariage de l'écriture et de l'action, Caillois ne relève cependant pas à quel point ses développements contenaient en eux-mêmes leur propre négation. La métaphore hivernale et glaciaire qu'il emploie de manière récurrente à propos de l'activisme du Collège trahit en effet l'irréalité de son projet politique et moral : censée manifester une toute-puissance éternelle de la pensée, elle renvoie aussi au refus de toute dépense et donc de toute action réelle. À la suite des travaux de l'école française de sociologie, Caillois insiste dans L'Homme et le sacré sur la nature ambivalente parce que sacrée du pouvoir auquel s'attache une géographie sociale du pur et de l'impur. On observe, d'un côté, « la clarté et la sécheresse du jour », "l'orient et le midi », «le haut» et «la droite»; de l'autre, «les ténèbres et 
l'humidité de la nuit», «le couchant et le nord», «le bas » et «la gauche ${ }^{37}$. Le premier aspect trouve sa transcription sociale et politique dans le monde majestueux et ordonné du roi, du prêtre et de la loi, tandis que le second se manifeste dans le domaine louche et infamant du paria et du coupable. Dans les textes publiés dans le cadre du Collège, Caillois oppose bien l'ordre du pouvoir et le désordre de la transgression, mais il échange en revanche les caractérisations solaires et estivales du premier contre celles, glaciaires et hivernales, du second. On se souvient qu'à la fin du «Vent d'hiver », il parle ainsi d' « un vent froid, rigoureux, arctique » ${ }^{38}$. Il présente ailleurs explicitement « la confrérie » comme une « organisation d'hiver ${ }^{39}$.

Ce n'est pas que la communion soit cependant envisagée par les sociologues français que Caillois a pu lire comme essentiellement hivernale. Certes Mauss montre que l'hiver est la saison privilégiée de la communion sociale dans les sociétés arctiques et Granet explique que la vie sociale est commandée en Chine par une alternance saisonnière dans laquelle l'hiver voit la régénération des forces Yang emprisonnées par les forces adverses du Ying ${ }^{40}$. Mais Caillois aurait pu s'inspirer d'autres textes mettant en avant la chaleur et non le froid de la communion, comme celui dans lequel Durkheim explique que, par-dessous « le froid moral qui règne à la surface de notre vie collective », la société est animée en profondeur par «des sources de chaleur» se formant «dans les classes populaires ${ }^{41}$ et pouvant être le creuset d'une nouvelle religion de l'homme.

En délaissant la chaleur de la communion socialiste et démocratique durkheimienne et en cherchant son inspiration chez Mauss et Granet, Caillois favorise une représentation hivernale du pouvoir qui contient en elle-même la négation de son efficience. L'immobilité glaciaire retient, en même temps qu'elle les manifeste, toute forme d'action. Le Collège est un foyer d'énergies froides qui ne se consument pas, dont le rayonnement est figé dans une dépense sous contrôle et sans déperdition. Cette dépense, qui s'apparente paradoxalement à une rétention, manifeste la toute-puissance du rayonnement narcissique, en même temps qu'elle le préserve de toute actualisation véritable. Caillois en expose le principe lui-même :

37. Ibid., p. 55 .

38. Roger Caillois, « Le vent d'hiver », op. cit., p. 352.

39. Roger Caillois, «Confréries, ordres, sociétés secrètes, églises », conférence du samedi 19 mars 1938, reprise in Denis Hollier, Le Collège de Sociologie (1937-1939), op. cit., p. 233.

40. Voir respectivement Marcel Mauss, «Essai sur les variations saisonnières des sociétés Eskimos », [Annales sociologiques, t. IX, 1904-1905], Sociologie et anthropologie, op. cit., p. 470 ; Marcel Granet, La Civilisation chinoise, [1929], Paris, Albin Michel, 1948, p. 228.

41. Émile Durkheim, «L'avenir de la religion », [séance du 18 janvier 1914 de l' «Union de libres penseurs et de libres croyants pour la Culture morale »], La Science sociale et l'action, [1970], Paris, PUF, 1987, p. 313. 
Comme un avare, caressant sa cassette, se paie de toutes ses privations à l'idée qu'il dispose, sous ses haillons, des richesses et du pouvoir d'un roi, [le membre de la secte] se flatte de mettre en branle à son gré une puissance que d'être mystérieuse fait paraître sans bornes, et non pas incertaine. ${ }^{42}$

L'avare ne manifeste pas sa puissance en dépensant son argent mais il la garde en réserve. L'occultation du trésor énergétique fait tout son prix. Caillois peut bien appeler de ses vœux un «nouveau printemps » ${ }^{43}$ à la fin du «Vent d'hiver », c'est contre cette dépense, cette rupture d'équilibre, contre l'instabilité d'un échange entre le monde et lui-même, c'està-dire finalement contre le principe de réalité, qu'il fige sa démarche dans un éternel hiver aussi glorieux qu'impuissant et, finalement, dépressif, comme en témoigne l'examen de conscience intitulé « Seres del anochecer » qu'il publie en espagnol dès décembre 1940 et qui constate terme à terme l'impossibilité d'atteindre la communauté idéale, dense et froide, forte et incorruptible, que devait former le Collège.

À sa manière si particulière, la sociologie active du Collège est le rêve d'un esprit, d'un poète et d'un intellectuel français, rêve surdéterminé donc - Caillois aimait le mot - en ce qu'il nie une triple réalité : celle d'un homme qui crut en la toute-puissance de sa pensée ; d'un poète qui imagina remplacer la poésie par la science comme d'autres immédiatement avant lui pensèrent remplacer la littérature par la poésie ; d'un intellectuel qui voyait fléchir les forces de la nation et imaginait que se placer sur le seul plan moral pourrait suffire à la relever. Fruit étrange de l'idéalisme foncier de la tradition sociologique durkheimienne et s'inscrivant dans la tradition française du poète romantique illuminant la société de ses visions, il ne débouche pas sur l'exercice réel d'un pouvoir mais sur la prétention illusoire qu'une idée du pouvoir aurait la capacité de s'imposer par son seul rayonnement. Au lieu de quoi, en juin 1940 l'hiver était loin —, les Allemands entrent dans Paris et la France se trouve clairement reléguée au rang de nation secondaire. Le Collège a échoué à atteindre les buts qu'il s'était fixé, il n'a pas non plus marqué l'histoire de la discipline et, à tout prendre, s'il jouit d'un certain prestige, c'est essentiellement au sein des études littéraires. Sans doute parce que l'impasse dans laquelle il s'est engouffré, si elle s'éloigne comme le siècle passé, ne cesse néanmoins d'attirer le regard et d'éveiller la nostalgie. Elle témoigne en effet d'un temps où les poètes osaient ce qui semble aujourd'hui l'impossible et où surtout la société leur reconnaissait encore ce droit ou consentait du moins à discuter avec eux : franchir les barrières disciplinaires, s'immiscer au sein du débat politique, occuper le cœur de la vie sociale — se battre avec énergie contre cet adieu à la littérature auquel semblent consentir aujourd'hui nos sociétés. De la même

42. Roger Caillois, L'Esprit des sectes, [1945], repris in Instincts et société. Essais de sociologie contemporaine, Paris, Denoël-Gonthier, 1964, p. 94.

43. Roger Caillois, «Le vent d'hiver », op. cit., p. 353. 
manière que le récit fantastique met en scène, comme Caillois l'explique lui-même, «un scandale, une déchirure, une irruption insolite, presque insupportable dans le monde réel ${ }^{44}$, le Collège de Sociologie met à mal la dichotomie contemporaine entre les discours savants et une littérature désormais considérée essentiellement comme un loisir. Il nous plonge du même coup dans l'inquiétude et nous pousse à nous interroger sur les modalités de nos écritures — ou sur leur absence. Il nous situe. 